\title{
ESTUDO DO LINFONODO SENTINELA NO CÂNCER DO COLO UTERINO COM AZUL PATENTE
}

\author{
Sabas Carlos Vieira*, luiz Carlos Zeferino, Benedito Borges da Silva, Jerúsia O. Ibiapina de Santana, \\ Lina Gomes dos Santos, Teresinha Castello Branco Carvalho, Maria Castelo Branco de Deus Rocha \\ Trabalho realizado no Departamento de Clínica Geral da Universidade Federal do Piauí e Hospital \\ São Marcos - Sociedade Piauiense de Combate ao Câncer, Teresina, PI.
}

\begin{abstract}
RESUMO - OBjetrvo. 0 objetivo deste estudo foi avaliar a identificação do linfonodo sentinela em pacientes com carcinoma do colo uterino submetidas a histerectomia radical e linfadenectomia pélvica através do mapeamento linfático com o corante azul patente.

Métodos. Cinquenta e um pacientes com carcinoma do colo uterino estádio I e II da Figo submeteram-se a histerectomia radical e linfadenectomia pélvica. $\mathbf{O}$ corante azul patente foi injetado em torno do tumor antes do início da cirurgia. No momento da linfadenectomia pélvica, os linfonodos claramente corados foram separados do restante do espécime da linfadenectomia.

Resultados. Em 32 das 51 pacientes foram identificados
\end{abstract}

linfonodos sentinela. Foram observados quatro casos de falso negativo. A especificidade foi de $38,6 \%$, a sensibilidade $42,8 \%$, o valor preditivo positivo $10 \%$ e o valor preditivo negativo foi de $80.9 \%$.

Conclusóes. A identificação do linfonodo sentinela em câncer do colo uterino é exeqüível com o corante azul patente. Entretanto, um estudo com maior número de casos é necessário para estabelecer a validade do conceito do linfonodo sentinela em câncer do colo uterino.

UnItermos: Colo uterino. Linfadenectomia. Biópsia de linfonodo sentinela.

\section{INTRODUÇÃO}

O linfonodo sentinela é o primeiro linfonodo regional a ser comprometido por metástases nas neoplasias malignas. Quando o linfonodo sentinela é negativo, os demais linfonodos da cadeia linfática também são negativos, com uma baixa incidência de falsonegativo. Portanto, o linfonodo sentinela é representativo do status linfonodal. Atualmente, a técnica da linfadenectomia sentinela é aplicada em melanoma e rapidamente está se tornando padrão também em câncer de mama, evitando as linfadenectomias desnecessárias e suas conseqüentes complicações. A identificação do linfonodo sentinela tem sido realizada através de mapeamento com radioisótopos e ou utilização de corantes. A taxa de identificação do linfonodo sentinela em câncer de mama utilizando-se radio sótopos, corante ou ambos foi de $92 \%, 81 \%$ e $93 \%$, respectivamente!

As pacientes com carcinoma do colo uterino são boas candidatas ao estudo do linfonodo sentinela por várias razões. Primeiro, a ocorrência de metástase pélvica nos

\section{* Correspondência:}

Rua 06, n²106 - Condomínio Tropical Park Bairro Santa Lia - CEP: 64055-150 - Teresina - PI sabasmarcia@uol.com.br estádios I e II varia de I | \% a 19\% e 24,5\% a $31 \%$, respectivamente 2 . Portanto, até $90 \%$ das pacientes com tumores pequenos podem ser submetidas a linfadenectomia pélvica desnecessariamente e ainda apresentar as complicações decorrentes da linfadenectomia, tais como lesão vascular, formação de linfocisto, lesão nervosa, trombose venosa, linfedema crônico de membros inferiores e aumento do tempo cirúrgico. Segundo, o colo uterino apresenta uma drenagem linfática complexa e uma extensa dissecção pélvica é necessária para remoção de todos os linfonodos. Outro aspecto é que a localização dos linfonodos comprometidos não pode ser determinada somente pela avaliação clínica e de exames de imagem. Finalmente, a identificação de pacientes com linfonodo positivo evita a cirurgia radical, selecionando-as para tratamento exclusivo com radio e quimioterapia.

Alguns estudos abordando o conceito de linfonodo sentinela em câncer do colo uterino têm sido publicados ${ }^{3-11}$. Entretanto conclusões definitivas ainda não estão disponíveis, pois o número de casos estudados até o momento ainda é pequeno.

O propósito do presente estudo foi avaliar a identificação do linfonodo sentinela em câncer do colo uterino utilizando-se o corante azul patente.

\section{Métodos}

De janeiro de 2000 a julho de 2002, 51 pacientes submetidas a histerectomia radical por carcinoma invasivo do colo do uterino foram elegíveis para o estudo. As pacientes incluídas no estudo pertenciam ao estadiamento la2,|b|,|b2 e II da FIGO. Todas as pacientes assinaram consentimento pós-informado. O estudo foi aprovado pelo comitê de ética e pesquisa da Universidade Federal do Piauí. Foram excluídas as pacientes submetidas previamente a radioterapia, quimioterapia e pacientes com história de alergia.

Após o ato anestésico, as pacientes foram posicionadas em posição de litotomia e o colo foi visualizado através de um espéculo vaginal. Foi injetado $2 \mathrm{ml}$ do corante patent blue 2,5\% (Guerbet S.A France) no colo uterino utilizando-se uma agulha de raquianestesia 25 - gauge. A injeção foi às $3,6,9$ e 12 horas na mucosa e estroma próximo ao tumor. A injeção do corante dentro do tumor foi evitada sempre que possível para diminuir o extravasamento do corante.

Após a injeção do corante, as pacientes foram colocadas em posição de decúbito dorsal para início da cirurgia. Realizou-se incisão abdominal mediana infra-umbilical ou transversa. Após a abertura da cavidade abdominal, observou-se as áreas coradas e se 
linfonodo corado (sentinela) era identificado este era dissecado e identificado de acordo com a área topográfica em que estava situado. Na ausência de identificação de linfonodos sentinela, procedeu-se a linfadenectomia pélvica bilateral com os seguintes limites: superior representado pelo cruzamento do ureter na artéria ilíaca comum; inferior origem da artéria epigástrica inferior; lateral nervo ileoinguinal; medial ureter e posterior o nervo obturador.

Todos os linfonodos sentinela foram submetidos a exame anatomopatológico de congelação no intra-operatório e também em parafina no pós-operatório para se estabelecer a concordância entre ambos.

Após o término da linfadenectomia, realizou-se histerectomia com parametrectomia e colpectomia (terço superior). Nas pacientes com linfonodo sentinela positivo no exame de congelação, a cirurgia prosseguiu com a realização da linfadenectomia e histerectomia com parametrectomia, pois esta é a conduta adotada na instituição.

Os linfonodos não corados foram submetidos a exames histopatológicos, através da coloração com hematoxilina e eosina. A sensibilidade, especificidade, valor preditivo negativo e positivo do linfonodo sentinela foram calculados.

\section{Resultados}

A idade das pacientes variou de 28 a 70 anos, com uma média de 46 anos. $O$ tipo histológico mais freqüente foi o carcinoma epidermóide com 43 casos (84,3\%). Em 39 pacientes $(76,5 \%)$ o estadiamento foi lb I e em oito (I $5,7 \%)$ foi lb2. O tempo de identificação do linfonodo sentinela após a injeção do corante no colo uterino variou de 20 a 83 minutos, com uma média de 45 minutos. Das 5 I pacientes, 32 (62,8\%) apresentaram linfonodos sentinela, totalizando 66 linfonodos, variando de I a 7 linfonodos, com média de 2, I linfonodos corados/paciente. A localização mais freqüente dos linfonodos sentinela foi a cadeia linfática da artéria ilíaca externa (Tabela I). Das 19 pacientes $(37,2 \%)$ que não apresentaram linfonodos sentinela, duas apresentaram linfonodos metastáticos no exame de parafina, portanto falso negativo.

Das 32 pacientes que apresentaram linfonodos sentinela, três tinham linfonodos sentinela metastáticos no exame de congela-

\begin{tabular}{lc}
\hline \multicolumn{2}{c}{ Tabela I - Localização dos 66 linfonodos sentinela } \\
\hline Cadeia linfática & Número (\%) \\
\cline { 2 - 2 } Ilíaca externa esquerda & $23(34,8)$ \\
Ilíaca externa direita & $19(28,8)$ \\
Ilíaca interna direira & $7(10,7)$ \\
Fossa obturadora direita & $4(6,1)$ \\
Ilíaca comum direita & $4(6,1)$ \\
Fossa obturadora esquerda & $3(4,5)$ \\
Parametrial direita & $3(4,5)$ \\
Ilíaca comum esquerda & $\mid(1,5)$ \\
Parametrial esquerda & $\mid(1,5)$ \\
Ilíaca interna esquerda & $\mid(1,5)$ \\
Total & $\mathbf{6 6}(\mathbf{1 0 0 \% )}$ \\
\hline
\end{tabular}

ção no intra-operatório. Entretanto, duas destas pacientes apresentaram também cada uma um linfonodo metastático não corado no exame anatomopatológico final de parafina. Os linfonodos sentinela que apresentaram metástase no exame de congelação também foram positivos no exame de parafina.

Das 29 pacientes em que o exame de congelação do linfonodo sentinela foi negativo, duas apresentaram linfonodos metastáticos não corados no exame de parafina (falso negativo). O número total de linfonodos ressecados foi de 956, variando de 8 a 34, com uma média de 18,7 linfonodos/paciente.

A especificidade, sensibilidade, valor preditivo positivo e negativo foram respectivamente de $39,6 \%, 100 \%, 9,4 \%$ e $100 \%$ para o exame de congelação. No exame histopatológico de parafina, a especificidade foi de $38,6 \%$, a sensibilidade $42,8 \%$, o valor preditivo positivo $10 \%$ e o valor preditivo negativo foi de $80,9 \%$.

\section{Discussão}

No presente estudo, identificou-se linfonodos sentinela em $62,8 \%$ das pacientes. Linfonodos pélvicos metastáticos ocorreram em sete $(13,7 \%)$ pacientes, das quais duas não apresentaram linfonodos sentinela (falso-negativo). Das 32 pacientes que apresentaram linfonodos sentinela, duas também tiveram metástase em linfonodos não-corados (falso-negativo).

O estudo do linfonodo sentinela em câncer do colo uterino foi originalmente descrito por Echi et al em 1999 (4). Estes autores utilizaram o Lymphazurin (issos sulfan blue), injetado diretamente no colo uterino de pacientes submetidas a histerec- tomia e linfadenectomia pélvica e paraórtica para tratamento do carcinoma do colo uterino. Foram estudadas 13 pacientes. Das três pacientes que apresentaram metástase linfonodal, duas tinham linfonodo sentinela. As dez pacientes restantes não apresentaram linfonodos sentinela.

A identificação do linfonodo sentinela em câncer do colo uterino tem variado na literatura de $15,3 \%$ a $100 \%{ }^{3-1}$. Esta variação tem sido decorrente das técnicas utilizadas, volume de corante injetado e número de pacientes estudadas. Um estudo encontrou que o volume de corante injetado foi determinante na taxa de sucesso na identificação dos linfonodos corados. Quando foi utilizado I,5ml de azul patente, a taxa de identificação de linfonodo corado foi de $50 \%$. Quando o volume foi aumentado para 2 a 3,9ml e maior que $4 \mathrm{ml}$, a taxa de linfonodos corados aumentou respectivamente para $83 \%$ e $92 \% 8$. A utilização de $0,2 \mathrm{~m}$ de azul patente em 10 pacientes com câncer do colo uterino foi capaz de identificar linfonodos sentinela em somente quatro casos $(40 \%)^{7}$.

Outro fator encontrado, segundo O'Boyle et $a l^{6}$, foi o tamanho do tumor do colo uterino, encontrando diferenças estatisticamente significantes na taxa de detecção de linfonodos corados quando compararam tumor maior ou menor que $4 \mathrm{~cm}$.

O tempo entre a injeção do corante no colo uterino e o aparecimento de canais linfáticos pélvicos corados é cerca de 5 a 10 minutos ${ }^{6}$. Em nosso estudo, todas as pacientes apresentavam canais linfáticos pélvicos corados e o tempo médio entre a injeção do corante e o encontro de linfonodos corados foi 45 minutos. 
Em um estudo com 26 pacientes ${ }^{8} \mathrm{com}$ carcinoma do colo uterino submetidas a tratamento cirúrgico e estudo do linfonodo sentinela com azul patente, a sensibilidade, o valor preditivo negativo e a acurácia foram de $100 \%$.

Outro autor ${ }^{5}$, usando tecnésio 99 e, ou azul patente $(2 \mathrm{ml})$, estudou 50 pacientes com carcinoma do colo uterino para determinar a validade do conceito de linfonodo sentinela em câncer do colo uterino. As pacientes pertenciam ao estádio I (32 pacientes), II (I6 pacientes), e IV (dois pacientes) da FIGO. As pacientes submeteram-se a histerectomia radical. Dez pacientes apresentaram metástase pélvica. Em 10 pacientes que não apresentaram linfonodo sentinela, quatro apresentaram metástase linfonodal. A taxa de detecção de linfonodo sentinela foi de $78 \%$. A sensibilidade e valor preditivo negativo foram $83,3 \%$ e $97,1 \%$, respectivamente. A taxa de falso negativo foi de $16,6 \%$.

O uso do tecnésio para identificação do linfonodo sentinela em câncer do colo uterino poderá diminuir a taxa de insucesso, como demonstraram Lantzsch et al. ${ }^{9}$ que não encontram nenhum falso negativo com este método. Dados corroborados por Levenbach et al. ${ }^{10}$ que estudaram 39 pacientes com câncer do colo uterino utilizando tecnesio e corante azul e encontraram sensibilidade de $87,5 \%$ e valor preditivo negativo de $97 \%$. Achados semeIhantes foram encontrados por Rhim et al. " que obtiveram valor preditivo positivo de $95,2 \%$ em 26 pacientes estudadas com tecnesio e issosulfan. Neste estudo, todas as pacientes foram submetidas a histerectomia radical e linfadenectomia pélvica e paraórtica.

Neste estudo, quatro pacientes apresentaram falso negativo para o linfonodo sentinela $(7,8 \%)$, ou seja, apresentaram metástase linfonodal pélvica e, portanto, o linfonodo sentinela não foi capaz de determinar o status linfonodal pélvico. A taxa de falso negativo da técnica da linfadenectomia sentinela em câncer de mama tem variado de $5 \%$ a $9 \%$ ! Como o número de pacientes com câncer do colo uterino estudados com esta técnica ainda é pequeno, é possível que a taxa de falso negativos diminua e que este procedimento venha a tornar-se padrão em câncer do colo uterino.
Outro aspecto estudado em câncer de mama é o valor da micrometástase detectada por imunoistoquímica no linfonodo sentinela. Os estudos iniciais não mostram impacto na sobrevida das pacientes que apresentam micrometástase e, portanto, a linfadenectomia nestes casos é controversa!. Estudos em câncer do colo uterino com relação a este aspecto não têm sido publicados.

Portanto, a identificação do linfonodo sentinela em câncer do colo uterino ocorreu em $62,8 \%$ dos casos. Entretanto, estudo com maior número de casos precisam ser realizados para que conclusões definitivas a cerca do conceito de linfonodo sentinela possam ser aplicadas em câncer do colo uterino.

\section{Conflito de interesse: não há.}

\section{SUMMARY}

\section{Sentinel node study with patent BLUE IN CERVICAL CANCER}

OBIETIVE. The purpose of this study was to determine the feasibility of sentinel node identification in patients with invasive cervix cancer undergoing radical hysterectomy and pelvic lymphadenectomy using intraoperative lymphatic mapping with patent blue dye.

METHODS. Fifty one patients wtih Figo I and II cervical cancer were submitted to radical hysterectomy and pelvic lymphadenectomy. The patent blue $V$ was injected around the tumor before beginning surgery. At the time of pelvic lymphadenectomy, lymph nodes with obvious uptake of the dye were separated from the remainder of the lymphadenectomy specimen.

RESULTS. Sentinel nodes were detected in 32 of the $5 \mathrm{I}$ patients. Four cases of false negative were observed. The specificity, sensitivity, positive predictive value and negative predictive value were $38.6 \%, 42.8 \%, 10 \%$ and $80.9 \%$, respectively.

ConCLUsIONs. Identification of sentinel node with patent blue dye is feasible in cervical cancer. A larger series will be required to establish sentinel detection in cervical cancer for further therapy concepts and planning. Rev Assoc Med Bras 2004; 50(3): 302-4]

KEY wORDS: Cervix uteri. Lymphadenectomy. Sentinel lymph node biopsy.

\section{REFERÊNCIAS}

I. Bonnema J, Van de Velde CJH. Sentinel lymph node biopsy in breast cancer. Ann Oncol 2002; | 3:153|-7.

2. Hatch KD. Cervical cancer. In: Berek JS, Haker Nf, editors: Practical gynecologic oncology. Baltimore: Williams and Wilkin; 1994. p.243.

3. Medl M, Peters-Engl C, Schutz P, Vesely M , Sevelda P. First report of lymphatic mapping with Isosulfan Blue Dye and sentinel node biops in cervical cancer. Anticancer Res 2000; 20: I 133-4.

4. Echt ML, Finan MA, Hoffman MS, Kline RC, Roberts WS, Florica JV. Detection of sentinel lymph nodes with lymphazurin in cervical, uterine, and vulvar malignancies. South Med Surg 1999; 92:204-8.

5. Malur S, Krause N, Köhler C, Schneider A. Sentinel lymph node detection in patients with cervical cancer. Gynecol Oncol 2001; 80:254-57.

6. O'boyle JD, Coleman RL, Bernstein SG, Lifshitz S, Muller CY, Miller DS Intraoperative lymphatic mapping in cervix cancer patients undergoing radical hysterectomy: A pilot estudy. Gynecol Oncol 2000; 79:238-43.

7. Verheijen RHM, Pijpers R, Van Diest PJ, Burger CW, Buist MR, Kenemans P. Sentinel Node Detection in cervical cancer. Obstet Gynecol 2000; 96:135-8.

8. Dargent D, Martin X, Roy M, Mathever P. Laparoscopic assessment of the sentinel lymph node in early stage cervical cancer. Gynecol Oncol 2000; 79(3):4I I-5.

9. Lantzsch T, Wolters M, Grimm J, Mende T, Buchmann J, Sliutz G, et al. Sentinel node procedure in lb cervical cancer: a preliminary series. $\mathrm{Br} J$ Cancer 2001; 85:791-4.

I0. Levenbach C, Coleman RL, Burke TW, Lin WM, Erdman W, Deavers M, et al. Lymphatic mapping and sentinel node identification in patients with cervix cancer undergoing radical hysterectomy and pelvic lymphadenectomy. J Clin Oncol 2002; 20:688-93.

I I. Rhim CC, Park JS, Bae SN, Namkoong SE. Sentinel node biopsy as na indicator for pelvic node dissection in early stage cervical cancer. J Korean Med Sci 2002; 17:507-।1.

Artigo recebido: 13/05/2003

Aceito para publicação: 05//2/2003 